\title{
Laparoscopic transverse colectomy using a new articulating instrument
}

\author{
Chul Seung Lee, Youngmin Kim, Yoon Suk Lee \\ Division of Colorectal Surgery, Department of Surgery, Seoul St. Mary's Hospital, College of Medicine, The Catholic University of Korea, Seoul, Korea
}

Laparoscopic complete mesocolic excision is the preferred approach for treating transverse colon cancer. Due to the anatomical complexity, mobilization and resection of the transverse colon can be technically challenging. This video demonstrates laparoscopic transverse colectomy using an articulating laparoscopic instrument for a 76-year-old female patient diagnosed with T-colon cancer.

Keywords: Articulating instrument, ArtiSential, Colonic neoplasms, Laparoscopy
Received June 25, 2021

Revised 1st August 5,2021 2nd August 25, 2021

Accepted September 3, 2021

Corresponding author

Yoon Suk Lee

Division of Colorectal Surgery,

Department of Surgery, Seoul St.

Mary Hospital, College of Medicine,

The Catholic University of Korea,

222 Banpo-daero, Seocho-gu, Seoul

06591, Korea

Tel: +82-2-2258-6095

Fax: +82-2-595-2282

E-mail: yslee@catholic.ac.kr

ORCID:

https://orcid.org/0000-0002-1849-2774

Copyright (๑) The Korean Society of Endoscopic and Laparoscopic Surgeons.
This is an Open Access article distributed under the terms of the Creative Commons Attribution Non-Commercial License (http:// creativecommons.org/licenses/by-nc/4.0/) which permits unrestricted non-commercial use, distribution, and reproduction in any medium, provided the original work is properly cited.

\section{INTRODUCTION}

The proximal two-thirds of the transverse colon is derived from the midgut, while the distal one-third is derived from the hindgut. They are supplied by the middle and left colic arteries, respectively [1]. From an anatomical perspective, the transverse colon is proximal to the upper abdominal vital structures and is not fixed to the retroperitoneal space [2].

Laparoscopic complete mesocolic excision (CME) is the preferred treatment option for transverse colon cancer due to its positive effect in terms of short-term morbidity, length of stay, and oncological outcome [2]. Due to the anatomical complexity, mobilization and resection of the transverse colon can be technically challenging. Manipulating tissues and obtaining the optimal angle are difficult with conventional laparoscopic instruments.

A robotic surgical system [3], which offered multi-jointed instruments, enhanced ergonomics, and three-dimensional vision, was developed to overcome these limitations. However, robotassisted surgery is considerably more expensive than laparoscopic surgery [4]. Several laparoscopic jointed instruments have been proposed as alternatives to the costly robotic systems [5]. In this video, we present a laparoscopic transverse colon resection performed using a laparoscopic articulating instrument (Supplementary Video 1). 


\section{METHODS}

The articulating laparoscopic instruments (ArtiSential; LIVSMED, Inc., Seongnam, Korea) were approved as class I medical devices by the Food and Drug Administration of Korea in 2019. The patient was a 76-year-old female with a body mass index of $22.2 \mathrm{~kg} / \mathrm{m}^{2}$. She was diagnosed with moderately differentiated adenocarcinoma of the mid-transverse colon. Initial computed tomography revealed clinical stage T3N1 without distant metastasis. Laparoscopic transverse colectomy was performed.

After endobronchial intubation, the patient was placed in a modified lithotomy position under general anesthesia. The 12$\mathrm{mm}$ trocars were placed in the umbilical position and left upper quadrant. An 8-mm trocar was placed in the left lower quadrant, while 5-mm trocars were placed in the right upper and lower quadrants. During the procedure, the surgeon used the ArtiSential instrument through the left upper and lower quadrant ports. The procedure was performed with bimanual manipulation using fenestrated forceps (ArtiSential) in the left hand and a monopolar hook (ArtiSential) in the right hand. ArtiSential fenestrated forceps are designed to deliver grasping of tissue through its atraumatic horizontal serrations and unique articulating technology. These articulating instruments allow the surgeon to easily move the laparoscope in any direction without obstructing the field of view. First, intraoperative indocyanine green (ICG)enhanced fluorescence was subjected to a subserosal injection to assess the appropriate dissection range. Using the 5-trocar approach, the middle colic artery and the colic branch of the gastrocolic trunk were ligated. After skeletalization of the middle and right colic vessels, vessel ligation was performed using a harmonic scalpel (Ethicon US, Somerville, NJ, USA) and 5-mm endo-clips (Endovision Co., Ltd., Daegu, Korea) followed by laparoscopic CME (Fig. 1). The colon was transected intracorporeally using an endoscopic stapler (Signia Stapling System; Covidien, Tokyo, Japan). Following intracorporeal side-to-side anastomosis

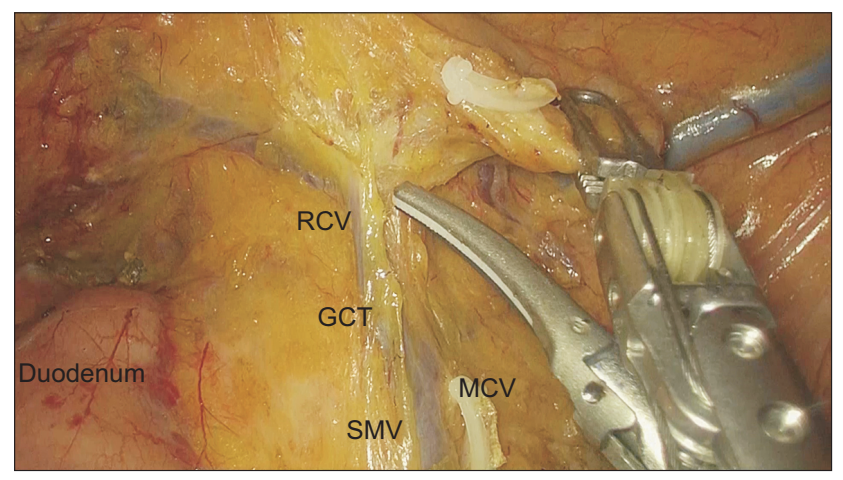

Fig. 1. Mesenteric lymphadenectomy. RCV, right colic vein; GCT, gastrocolic trunk; MCV, middle colic vein; SMV, superior mesenteric vein. using a Signia Stapling System, a continuous suture, using barbed thread, was made from the distal edge of the enterotomy to the proximal edge. Intracorporeal side-to-side anastomosis was performed after checking the perfusion status using ICG.

The specimen was placed in a specimen retrieval bag (Lapbag; Sejong Medical Corp., Paju, Korea), and the umbilical trocar site was extended to $5 \mathrm{~cm}$. Then, the specimen was removed from the abdominal cavity (Fig. 2). After the wound retractor was placed through a midline incision, a pneumoperitoneum was formed. After applying surgical glue to the anastomosis site, a Jackson Pratt drain $200 \mathrm{~mL}$ was inserted into the Douglas pouch using the left lower quadrant port.

\section{RESULTS}

The total operation time was 180 minutes with an estimated blood loss of $20 \mathrm{~mL}$. There were no perioperative complications, and the patient has prescribed a soft diet on postoperative day 1. The patient was discharged on postoperative day 3. The final pathological diagnosis was pT3N1bM0. There were 34 harvested regional lymph nodes with three identified metastatic nodes.

\section{DISCUSSION}

The disadvantages of using conventional laparoscopic instruments with fixed joints include reduced dexterity, limited range of movement, and uncomfortable ergonomics. Obtaining an effective angle, or performing traction and countertraction during laparoscopic surgery can be challenging. Although the robotic surgical system addresses these problems, the issue of cost-effectiveness persists [4]. The new laparoscopic articulating instruments (ArtiSential) help surgeons obtain effective traction and countertraction through intuitive movements. The articulating joints of the instruments are synchronized with the user's wrist

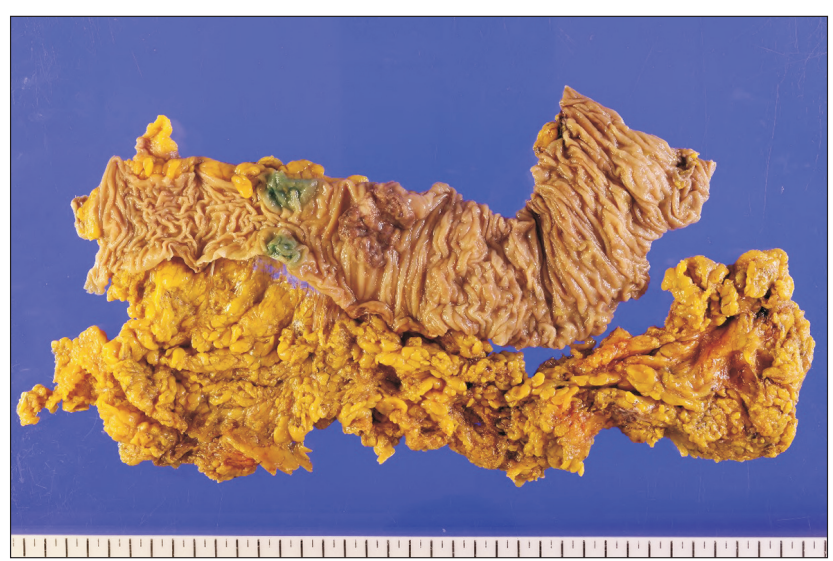

Fig. 2. Laparoscopic transverse colectomy specimen showing a ulcerofungating mass lesion of transverse colon. 
motion and can be moved by $360^{\circ}$, allowing for more versatile surgical procedures. Compared to the conventional laparoscopic devices, these new instruments make it easier to grab, lift, or apply traction to tissue in narrow spaces. However, its gripping force is weaker and less fixed than the conventional laparoscopic device. Hence, conventional straight instruments were used as assistive devices in the surgery. Moreover, the new instrument is larger and heavier than the conventional instruments. A smaller and lighter design will be more convenient for surgeons.

Unfortunately, in cases with bulky tumors, narrow operation fields, or obese patients, surgical procedures with conventional laparoscopic instruments are more technically demanding. In this video, we present a standardized procedure for laparoscopic transverse colectomy using two ArtiSential instruments. The use of the articulating laparoscopic instrument is particularly helpful for exposing surgical planes and skeletonizing the primary feeding vessels.

The surgical blood supply quality was assessed using ICG fluorescence angiography (FA). The intraoperative ICG FA was used to evaluate the perfusion of the anastomosis. A uniform green glow emission from the ICG injection indicated adequate perfusion to the anastomosed colon site.

This was the first video presenting the clinical application of the newly released articulating laparoscopic instrument (ArtiSential). Laparoscopic transverse colectomy using an articulating laparoscopic instrument is safe and technically feasible.

\section{NOTES}

\section{Ethical statements}

The Institutional Review Board of The Catholic University of Korea, Seoul St. Mary's Hospital approved this study and waived the requirement for informed consent (No. KC21ZISI0492), and we followed the principles of the Declaration of Helsinki for health research ethics.

\section{Authors' contributions}

Conceptualization: All authors

Data curation, Formal analysis: CSL

Investigation: YSL
Methodology: YK

Project administration: CSL, YSL

Visualization: SJK, YK

Writing-original draft: All authors

Writing-review \& editing: All authors

All authors read and approved the final manuscript.

\section{Conflict of interest}

All authors have no conflicts of interest to declare.

\section{Funding/support}

None.

\section{ORCID}

Chul Seung Lee, https://orcid.org/0000-0002-4859-3015

Youngmin Kim, https://orcid.org/0000-0001-8900-3854

Yoon Suk Lee, https://orcid.org/0000-0002-1849-2774

\section{Supplementary materials}

Supplementary materials can be found via https://doi.org/10.7602/ jmis.2021.24.4.227.

\section{REFERENCES}

1. Zmora O, Bar-Dayan A, Khaikin M, et al. Laparoscopic colectomy for transverse colon carcinoma. Tech Coloproctol 2010;14:25-30.

2. Storli KE, Eide GE. Laparoscopic complete mesocolic excision versus open complete mesocolic excision for transverse colon cancer: longterm survival results of a prospective single centre non-randomized study. Dig Surg 2016;33:114-120.

3. Park SY, Choi GS, Park JS, Kim HJ, Choi WH, Ryuk JP. Robotassisted right colectomy with lymphadenectomy and intracorporeal anastomosis for colon cancer: technical considerations. Surg Laparosc Endosc Percutan Tech 2012;22:e271-e276.

4. Baek SJ, Kim SH, Cho JS, Shin JW, Kim J. Robotic versus conventional laparoscopic surgery for rectal cancer: a cost analysis from a single institute in Korea. World J Surg 2012;36:2722-2729.

5. Lee CS, Lee YS. Laparoscopic low anterior resection using new articulating instruments. Tech Coloproctol 2021;25:1259-1261. 